\title{
Antithrombotic management after transcatheter aortic valve implantation
}

\author{
Fiachra McHugh $^{1}$, Khalid Ahmed ${ }^{1}$, Antoinette Neylon ${ }^{1}$, Faisal Sharif $^{1,2}$, Darren Mylotte ${ }^{1,2}$ \\ ${ }^{1}$ Department of Cardiology, University Hospital of Galway, Galway, Ireland; ${ }^{2}$ National University of Ireland, Galway, Ireland \\ Contributions: (I) Conception and design: D Mylotte; (II) Administrative support: None; (III) Provision of study materials: None; (IV) Collection and \\ assembly of data: None; (V) Data analysis and interpretation: None; (VI) Manuscript writing: All authors; (VII) Final approval of the manuscript: All \\ authors. \\ Correspondence to: Darren Mylotte, BCh, MB, MD. Department of Cardiology, University Hospital of Galway, Newcastle Road, Galway, Ireland. \\ Email: darrenmylotte@gmail.com.
}

\begin{abstract}
Transcatheter aortic valve implantation (TAVI) is now the accepted standard of care for patients with symptomatic severe aortic stenosis at elevated risk for conventional surgical valve replacement. Currently, societal guidelines propose the use of dual antiplatelet therapy for the prevention of thromboembolic events after TAVI in patients without an indication for oral anticoagulation. This strategy is empiric and largely based on expert consensus extrapolated from the arena of percutaneous coronary intervention. In this review, we explore the rational for using antiplatelet and/or anticoagulant strategies after TAVI, review current guidelines and the evidence underpinning them, and detail the on-going randomized trials that will shape future recommendations on this important issue.
\end{abstract}

Keywords: Transcatheter aortic valve implantation (TAVI); transcatheter aortic valve replacement (TAVR); antiplatelet therapy; anticoagulation; vitamin $\mathrm{K}$ antagonists (VKA); novel anticoagulants

Submitted Oct 02, 2018. Accepted for publication Oct 16, 2018.

doi: $10.21037 /$ jtd.2018.10.59

View this article at: http://dx.doi.org/10.21037/jtd.2018.10.59

\section{Introduction}

Transcatheter aortic valve implantation (TAVI) has emerged as a gold standard therapeutic option for patients with symptomatic severe aortic stenosis who are ineligible for conventional surgical aortic valve replacement (SAVR) or at high- or intermediate-operative risk (1). The expansion of this technology to younger and lower risk patients is under investigation and the number of patients treated with TAVI globally continues to increase at an exponential rate.

As with all transcatheter vascular interventions, TAVI exposes patients to a small but important risk of periprocedural thromboembolic complications including stroke, systemic embolization, and myocardial infarction (MI). These patients are at the same time at risk of procedural vascular complications and major bleeding events. Most adverse events occur peri-procedurally, however thromboembolic and bleeding events can occur far beyond the index intervention and significantly impact long-term outcomes.

A variety of antiplatelet and anticoagulant strategies are being used to offset the risk of thromboembolic complications after TAVI. Depending on the potency of the antiplatelet or anticoagulant drugs used, each therapeutic regimen will have a certain efficacy and safety profile. Striking the balance between embolic and bleeding events for a given patient can be challenging, especially among TAVI patients who are by definition at high-risk for both (Figure 1). To date, the optimal anticoagulant regimen after TAVI has not been established and there is a paucity of high-quality evidence to provide guidance on this important subject.

Herein, we review current practice patterns, guideline recommendations, clinical trial results and on-going research efforts with respect to antithrombotic therapy 


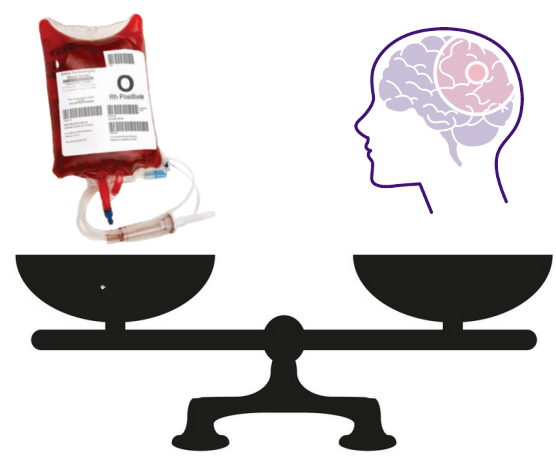

Figure 1 Balancing the risks of embolic and bleeding events after TAVI. TAVI, transcatheter aortic valve implantation.

after TAVI.

\section{Thromboembolic events after TAVI}

Stoke is among the most feared complications of TAVI. Approximately half of all cerebrovascular events occur within 24 hours of the TAVI procedure and can be attributed to wire and catheter manipulation, expansion of the transcatheter heart valve (THV) itself or the requirement for pre/post balloon dilatation $(2,3)$. Accordingly, debris extracted from embolic protection filters during TAVI includes aortic wall and atherosclerotic tissue, leaflet tissue and calcific deposits, myocardium, thrombus, and foreign material (4). This highlights multiple sites of endothelial injury during a TAVI procedure with the potential to create a nidus for embolic debris during or after the procedure.

Subacute (1-30 days) or late ( $>30$ days) stroke after TAVI may be a result of the interaction between the THV and the prothrombotic milieu of advanced atherosclerotic disease found in the TAVI population $(3,5)$. Thrombus development on or around the THV leaflets/frame may occur as a response to tissue factor release from the compressed native atherosclerotic leaflets or due to creation of pockets of slow and/or turbulent blood flow around the leaflets/frame which predisposes to fibrin deposition and thrombus formation (6). Aortic wall injury with subsequent healing and activation of the clotting cascade may also predispose to late embolic events after TAVI.

Atrial fibrillation (AF), which can occur in up to $30 \%$ of candidates, is the most common aetiology of late stroke after TAVI. New onset AF occurs in up to a third of patients undergoing TAVI and is also associated with an increased risk of cardioembolic events (7). Taken together, AF and the thrombotic environment of the aortic root are likely responsible for the observed elevated stroke/cardioembolic risk that persists for up to 3 months after valve replacement (8).

\section{Bioprosthetic leaflet thrombosis}

Bioprosthetic leaflet thrombosis is an important consideration when determining antithrombotic strategy after TAVI. This imaging entity occurs in approximately $13 \%$ of patients undergoing TAVI and appears to be valvespecific (9). The mechanism of leaflet thrombosis is not clearly understood but recent observations that thrombosis is more likely to occur with incomplete THV frame expansion or with leaflet overhang suggest that localized slow flow may create a prothrombotic environment (10). Other associated factors include concomitant cancer diagnosis and valvein-valve procedures (11). Leaflet thrombosis occurs more frequently in patients taking antiplatelet therapy versus oral anticoagulants (4\% vs. 15\%) (11).

Leaflet thrombosis encompasses a disease spectrum ranging from subclinical hypo-attenuating leaflet thickening (HALT) to clinically apparent valve thrombosis, with elevated transvalvular gradients and potentially early valve failure. Nearly one fifth of patients with THV thrombus go on to develop clinically overt obstructive valve thrombosis. At the lower end of the spectrum, subclinical leaflet thickening or immobility, has not been conclusively linked a higher risk of thromboembolic events, particularly stroke. In most cases, oral anticoagulation efficiently resolves HALT and hypo-attenuation affecting motion (12). A single report has suggested that leaflet thickening may be associated with an elevated risk of transient ischaemic attack, but adjudication of this "softer" endpoint can be challenging (11).

\section{Bleeding after TAVI}

Periprocedural life-threatening and major bleeding events after TAVI occur due to vascular complications or pericardial bleeding. Vascular access complications are the most common cause of early major bleeding events and gratifyingly, the incidence of these events has fallen due to improved operator experience, device iteration, the introduction of multislice computed tomography assessment of the peripheral vasculature, and enhanced patient selection. Vascular complications are however closely linked to bleeding events, and major bleeding conveys a considerable increase risk of 30-day and 1-year mortality, 
Table 12017 Antiplatelet \& anticoagulant recommendations after TAVI without alternate indication for oral anticoagulation

\begin{tabular}{lccc}
\hline Guideline & Single antiplatelet therapy & Dual antiplatelet therapy & Vitamin K antagonist \\
\hline 2017: AHA/ACC & $\times$ & $\sqrt{ }(\mathrm{llb}, \mathrm{C})$ & $\sqrt{ }(\mathrm{lllb}, \mathrm{B})$ \\
Duration & & 6 months & 3 months \\
Comment & $\sqrt{ }(\mathrm{llb}, \mathrm{C})$ & $\sqrt{ }(\mathrm{lla}, \mathrm{C})$ & Low bleeding risk \\
2017: ESC/EACTS & & $3-6$ months & $\times$ \\
Duration & High bleeding risk & & \\
Comment & & & \\
\hline
\end{tabular}

$\times$, not recommended; $\sqrt{ }$, recommended. TAVI, transcatheter aortic valve implantation; NOAC, novel oral anticoagulant; AHA, American Heart Association; ACC, American College of Cardiology; ESC, European Heart Association; EACTS, European Association for CardioThoracic Surgery.

irrespective of the aetiology of the bleeding $(13,14)$.

Late bleeding events ( $\geq 30$ days after TAVI) are also an important predictor of adverse outcome. In the PARTNER trial and continued access registry, late bleeding events occurred in $5.9 \%$ of cases at a median time of 132 days (interquartile range, 71-230 days) and were associated with a near 4-fold increased risk of death between 30 days and 1 year [hazard ratio (HR): $3.91 ; 95 \%$ confidence interval (CI): 2.67-5.71] (15). In this series, gastrointestinal (40.8\%), neurological $(5.5 \%)$, and traumatic falls $(7.8 \%)$ were the most frequent aetiology of bleeding.

Piccolo and colleagues reported bleeding events (lifethreatening, major, and minor) in $30 \%$ of patients at 5 years post TAVI; there was a similar proportion of access-site and non-access-site bleeding events, but nonaccess site bleeding conveyed a 1.5 times greater risk of all-cause mortality (16). The substantial impact of nonaccess site related bleeding is not surprising: we have witnessed the relevance of non-access site bleeding in the field of percutaneous coronary intervention (17). Bleeding risk is related to a variety of patient factors including age ( $\geq 75$ years), comorbid illnesses (renal or liver disease or cancer), bleeding history (intracranial haemorrhage, actionable bleeding), and laboratory indices (anaemia, platelet count), as well as medication regimen (oral anticoagulants, non-steroidal anti-inflammatory drugs).

These data confirm that bleeding events are clearly associated with adverse outcome, irrespective of timing or aetiology. The TAVI field has clearly focussed on reducing vascular complications and hence, access-site related bleeding. Further progress is however required to reduce the risk of non-access site bleeding in the acute and post discharge period. In this respect, the choice of antithrombotic regimen (duration and potency) can have a considerable impact.

\section{Societal guidelines}

The 2017 update of the valvular heart disease guidelines of the European Society of Cardiology/European Association of Cardio-Thoracic Surgery and the American Heart Association/American College of Cardiology propose different recommendations for antiplatelet or anticoagulant therapy after TAVI (Table 1) $(1,18)$. Both recommend dual antiplatelet therapy with aspirin and clopidogrel for between 3-6 (EU) or 6 (US) months and life-long aspirin thereafter. Only in the European guidelines is consideration is given to using single antiplatelet therapy (SAPT) (aspirin) in patients deemed to be at high bleeding risk. In the US guidelines, the use of a vitamin $\mathrm{K}$ antagonist (VKA) to offset the risk of leaflet thrombosis is suggested for patients at low bleeding risk. In both guidelines, continuation of oral anticoagulation alone is recommended in patients with an alternative indication for oral anticoagulation, such as $\mathrm{AF}$.

These recommendations are diminished by a paucity of clinical trial evidence evaluating different antiplatelet regimens after TAVI (Level of Evidence B or C). There is therefore great uncertainty in the TAVI community on the application of this guidance. A recent survey of 250 TAVI centres from 38 different countries reported highly variable practices post TAVI: DAPT of 1,3 , and $>/=6$ months duration was used in $14 \%, 41 \%$ and $32 \%$ of centres, respectively (19). There was also a lack of consensus in antithrombotic regimen in TAVI patients with AF: anticoagulation alone, anticoagulation with aspirin, and anticoagulation with clopidogrel, or triple therapy was used in $28 \%, 37 \%, 26 \%$ and $4 \%$ of centres, respectively. Evidence-based guidance is urgently required for the field. 


\section{The antiplatelet and antithrombin hypotheses}

The choice of an antiplatelet or anticoagulant strategy to prevent ischaemic events after TAVI is based on the assumption that these events are either caused by plateletor thrombin-mediated thrombosis, respectively. Currently, we have do not have a good mechanistic grasp of the underlying pathobiology of these post-TAVI events to guide the selection of post-implantation drug therapy. Moreover, the elderly TAVI population is complicated by the requirement for co-prescribing of antiplatelet or anticoagulant therapy in a large proportion of patients due to the high prevalence of coronary artery disease or AF.

Dual antiplatelet therapy (DAPT) has been the "thromboembolic-prevention" regimen of choice since the advent of TAVI but is not evidence-based. The antiplatelet hypothesis aims to prevent thromboembolic events by inhibiting the development of platelet-mediated thrombosis on the THV stent frame until endothelialisation occurs at approximately 3 months (20). Although DAPT reduces ischaemic events in the setting of coronary stenting, there is an associated increased risk of bleeding with prolonged DAPT. There is therefore considerable debate as to the relative benefit of reducing ischaemic events with DAPT with respect to the relative benefit of reducing in bleeding complications with SAPT.

The antithrombin hypothesis aims to prevent the development of thrombin-mediated clot on the leaflets or frame of the THV. Historically, a short course (3 months) of oral anticoagulation after implantation of an aortic surgical bioprosthetic valve had been advocated (8). More recently, societal guidelines have recommended aspirin monotherapy due to increased bleeding risk, unless another indication for anticoagulation is present $(1,18)$. The use of oral anticoagulants after TAVI is the subject of numerous on-going randomized trials, and will be discussed later in this review.

To date, it remains unclear if thromboembolic events after TAVI are principally caused by platelet- or thrombinmediated clot formation. Further basic science research is required to provide guidance to inform future clinical trial design on this topic.

\section{Antiplatelet hypothesis: available evidence}

Three completed randomized controlled trials have compared SAPT and DAPT after TAVI (21-23). First, Ussia and colleagues compared 3 months of DAPT (aspirin and clopidogrel) with aspirin monotherapy in a single centre study of 79 patients undergoing TAVI with a selfexpanding device (21). These authors found no betweengroup difference in the incidence of major adverse cardiac and cerebrovascular events at 6 months. The observed rates of stroke at 30-day were $3 \%$ and $5 \%$ in DAPT and aspirin groups, respectively. The small sample size of this study renders any conclusions as hypothesis-generating only.

Next, the SAT-TAVI (SAPT for TAVI) study randomized 120 patients to 6 months DAPT or aspirin only (22). This pilot study was also underpowered to detect differences in bleeding or ischaemic endpoints. These authors reported no between-group difference in the rate of 30-day major stroke rate (1.7\% in both groups) or VARC-defined 30-day safety endpoint. The rate of vascular complications was lower in the SAPT group ( $13 \%$ vs. $5 \% ; \mathrm{P}=0.03)$.

Finally, the ARTE (Aspirin Versus Aspirin + Clopidogrel Following Transcatheter Aortic Valve Implantation) trial compared 3 months of DAPT to aspirin monotherapy in 222 patients undergoing TAVI with a balloon-expandable device (study was scheduled to include 300 patients but was stopped prematurely due to slow recruitment and funding issues) (23). The primary endpoint was a composite of ischaemic (death, MI, stroke or transient ischemic attack) and major or life-threatening bleeding events at 3 months. Ischaemic events tended to occur more frequently in the DAPT group $(15.3 \%$ vs. $7.2 \%, \mathrm{P}=0.065)$. DAPT was also associated with a higher rate of major or life-threatening bleeding (10.8\% vs. $3.6 \%, \mathrm{P}=0.038)$.

A patient-level meta-analysis of the 421 patients included in these 3 studies found a similar rate of stroke (HR 1.00, 95\% CI: 0.29-3.48, $\mathrm{P}=1.00)$ and death (HR 1.40, 95\% CI: $0.56-3.51, \mathrm{P}=0.48)$ between DAPT and aspirin monotherapy (24). In contrast, there was a 3 -fold higher rate of life-threatening bleeding with DAPT compared to aspirin alone (HR 2.68, 95\% CI: 1.07-6.7, $\mathrm{P}=0.036$ ). Considered together, these data suggest that DAPT does not reduce the rate of ischaemic events after TAVI but significantly increases bleeding risk.

\section{Antiplatelet hypothesis: on-going trials}

As described above, the available evidence points to net clinical harm with DAPT compared to SAPT after TAVI. Several on-going important clinical trials are testing this hypothesis (Table 2).

The antiplatelet therapy for patients undergoing TAVI (POPular-TAVI) trial is a prospective randomized, 


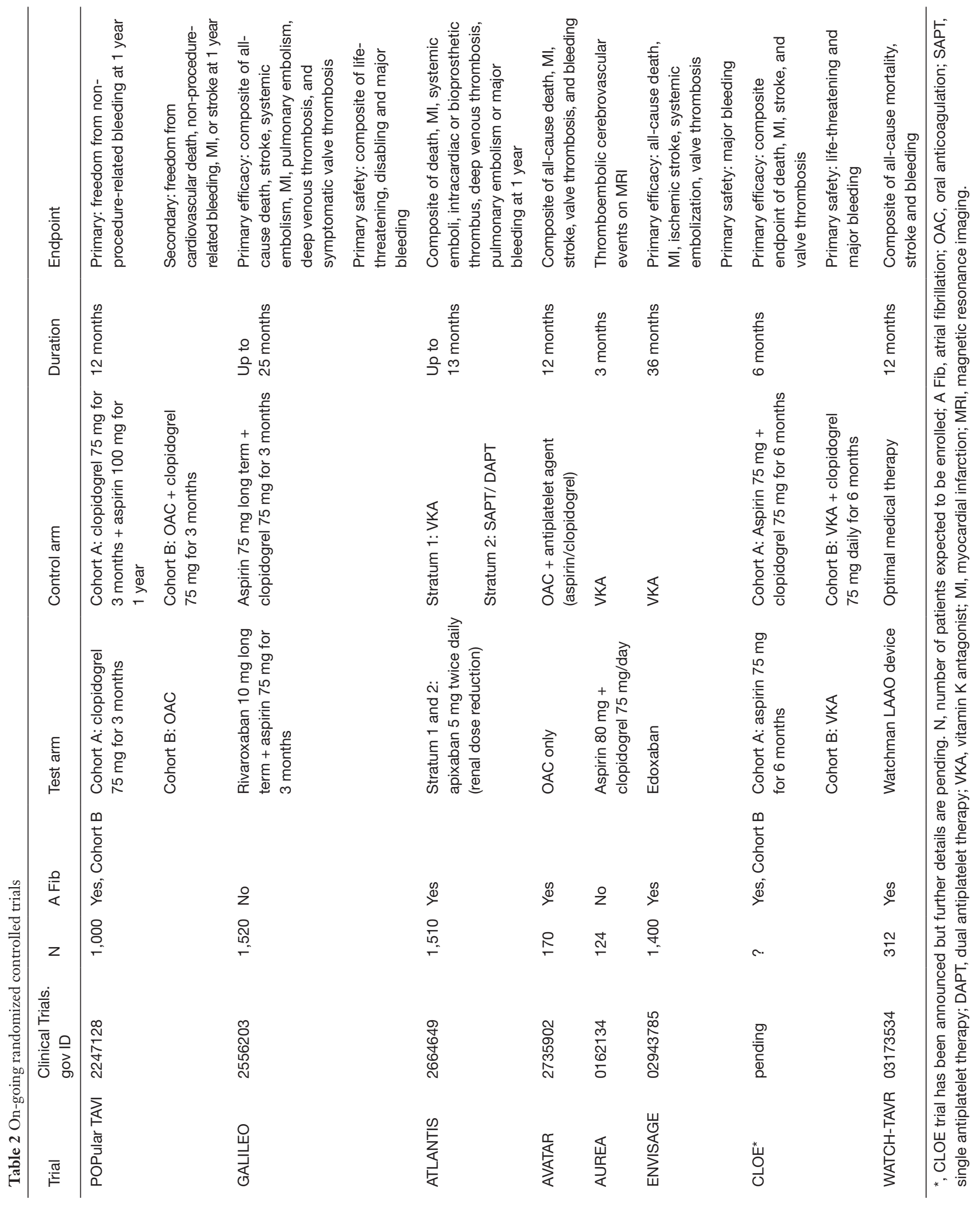


controlled, open-label multicentre clinical trial testing the hypothesis that monotherapy with aspirin or oral anticoagulation after TAVI is safer than the addition of clopidogrel for 3 months, without compromising clinical benefit (25). In this study, two cohorts are identified: in cohort A $(n=684)$, patients are randomized to aspirin monotherapy or DAPT for 3 months; in cohort B $(n=316)$, patients with an indication for oral anticoagulation are randomized to oral anticoagulation alone or anticoagulation in addition to clopidogrel. The primary outcome (superiority) is freedom from non-procedurerelated bleeding at 1 year. A secondary, net-clinical benefit outcome (non-inferiority) is freedom from the composite of cardiovascular death, non-procedural-related bleeding, MI, or stroke at 1 year. Recruitment began in February 2014 and is expected to finish in 2019.

The CLOE study has a similar design to POPular (two identical cohorts) except that the duration of the study drug is continued for 6 rather than 3 months. In CLOE, there are two co-primary endpoints: a primary efficacy composite endpoint of death, MI, stroke, and valve thrombosis; and a primary safety endpoint of VARC-defined life-threatening and major bleeding.

\section{Anticoagulation hypothesis: available evidence}

Currently, there are no completed randomized trials evaluating the safety and efficacy of oral anticoagulation after TAVI. Observational data on this subject should be considered to be hypothesis generating only.

A retrospective analysis including data from 12 centres compared outcomes in 621 patients with AF undergoing TAVI treated with either oral anticoagulation alone or oral anticoagulation and SAPT or DAPT (26). After a median follow-up of 13 months, the rates of death, stroke and MI were similar between groups. Those treated with oral anticoagulation plus antiplatelet therapy had a higher risk of major or life-threatening bleeding (24.4\% vs. $14.9 \%$, adjusted HR 1.85, 95\% CI: 10.5-3.28, P=0.004).

Demonstrating the trade-off between the potential benefits and harm of oral anticoagulation after TAVI, the FRANCE-TAVI registry has recently reported factors associated with long-term mortality and bioprosthetic valve dysfunction (27). These authors found that a history of AF, and thus anticoagulation, was the strongest independent correlate of mortality (adj. HR 1.41, 95\% CI: 1.23-1.62, $\mathrm{P}<0.001$ ), while anticoagulation at discharge (adj. odds ratio $0.54,95 \%$ CI: $0.35-0.82, \mathrm{P}=0.005$ ) was independently associated with lower rates of bioprosthetic valve dysfunction.

The introduction of novel oral anticoagulants (NOAC) has revolutionized the management of patients with AF. Compared to VKA, NOACs reduce mortality, cardiovascular mortality, stroke, and systemic embolism (28). Seeger and colleagues compared Apixaban to VKA in 272 TAVI patients with $\mathrm{AF}$ and found significantly lower rates of 30-day VARC-2 safety endpoints in the apixaban-treated cohort $(13.5 \%$ vs. $30.5 \%$; $\mathrm{P}<0.01)$ (29). The rates of stroke and bleeding were similar in both groups.

These data suggest that in TAVI patients with AF, oral anticoagulation alone should be the treatment of choice as additional antiplatelet therapy does not appear to reduce the risk of thromboembolic events but does markedly increase bleeding risk. The role of oral anticoagulation after TAVI in patients without $\mathrm{AF}$ is currently under investigation.

\section{Anticoagulation hypothesis: on-going trials}

Several randomized trials are testing the comparative safety and efficacy of oral anticoagulation with that of DAPT (Table 2). In POPular-TAVI, Cohort B will compare oral anticoagulation with oral anticoagulation and 3 months of clopidogrel in patients with AF after TAVI (25). The anticoagulation alone versus anticoagulation and aspirin following TAVI (AVATAR) study is an open label multicentre randomized comparison of the same study groups as Cohort B of POPular-TAVI. The dual antiplatelet therapy versus oral anticoagulation for a short time to prevent cerebral embolism after TAVI (AUREA) study aims to evaluate the effectiveness of DAPT versus oral anticoagulation for prevention of cerebral thromboembolism after TAVI. In this study, pre and post TAVI (3 months) magnetic resonance imaging will be compared to evaluate the primary endpoint of new areas of cerebral infarction.

Three other studies are evaluating the role of NOACs in thromboprophylaxis after TAVI. The global study comparing a rivaroxaban-based antithrombotic strategy to an antiplatelet-based strategy after transcatheter aortic valve replacement (TAVR) to optimize clinical outcomes (GALILEO) will compare 3 months of DAPT (then aspirin only for 18 months) to 3 months of rivaroxaban and aspirin (then rivaroxaban only for 18 months). The primary efficacy endpoint is a composite of death, MI, stroke, symptomatic valve thrombosis, systemic embolization or major venous thromboembolism. The primary safety endpoint is a 
composite of life-threatening, disabling and major bleeding. Embedded within the GALILEO design are two important substudies: the GALILEO-4D study (NCT 02833948) is a 4-dimensional computed tomography evaluation of the relative risk of developing subclinical leaflet thrombosis in both study arms (N=300); and the Evaluation of Cerebral Thromboembolism After TAVI (EARTH) study (NCT 02758964) will evaluate the occurrence and extent of cerebral embolization (total new lesion volume) in 180 study patients before and 3 months after TAVI. Most recently, it was announced that the data and safety monitoring board of the GALILEO trial have halted the study due to possible safety issues after an independent ad-hoc analysis of uncleaned data available on $31^{\text {st }}$ July 2018 , including clinical events not yet adjudicated and confirmed by the clinical events committee. Further information on this unexpected turn of events is eagerly awaited.

The anti-thrombotic strategy after trans-aortic valve implantation for aortic stenosis (ATLANTIS) study $(\mathrm{N}=1,510)$ is composed of two stratum: Stratum 1 compares VKA to Apixaban $5 \mathrm{mg}$ twice daily in patients with AF; and Stratum 2 compares SAPT or DAPT and Apixaban. Finally, the edoxaban compared to standard care after heart valve replacement using a catheter in patients with atrial fibrillation (ENVISAGE) trial will compare VKA and edoxaban $60 \mathrm{mg}$ in 1,400 patients after TAVI.

\section{The future}

Clearly, the results of the multiple on-going randomized trials will inform future guidelines and consensus documents regarding the optimal thromboprophylaxis strategy after TAVI. One potential alternate to long-term anticoagulation in patients with $\mathrm{AF}$ is left atrial appendage occlusion (LAAO). This strategy could play a role in patients with AF that have an absolute or relative contraindication to oral anticoagulation. A small study of 52 patients undergoing TAVI and simultaneous LAAO demonstrated the feasibility of this approach (30). The Watchman (Boston Scientific, Marlborough, MA, USA) for patients with atrial fibrillation undergoing transcatheter aortic valve replacement (WATCH-TAVR) trial is evaluating the safety and efficacy of this approach in 312 patients randomized to the Watchman device or optimal therapy.

Another area of current interest is the potential role that platelet function testing could play in identifying patients at high ischaemic or bleeding risk while taking DAPT. A recent analysis in 146 TAVI patients has suggested that low on-treatment platelet reactivity, determined by adenosine diphosphate-induced platelet aggregation with Multiplate, predicted bleeding events (adjusted HR 2.06, 95\% CI: 1.14-3.71, $\mathrm{P}=0.02$ ) (31). Perhaps this strategy could be used to individualize an antiplatelet therapy regimen for a given patient although this strategy has not proven to be effective in the realm of coronary interventions.

\section{Conclusions}

The routine use of DAPT after TAVI is empiric and not evidence-based. SAPT appears to cause less bleeding when compared to DAPT and may ultimately be the regimen of choice in those without another indication for DAPT or oral anticoagulation after TAVI. While oral anticoagulation has the potential to reduce bioprosthetic valve thrombosis, unadjudicated events in the GALILEO study suggest that this strategy may be associated with an excess of bleeding, without a commensurate reduction in ischaemic events. In patients with AF, a strategy of oral anticoagulation alone without an additional antiplatelet agent is the subject of current study. The innumerable on-going trials in this field will inform considerable guideline change in the coming years.

\section{Acknowledgements}

None.

\section{Footnote}

Conflicts of Interest: D Mylotte: Consultant and Proctor for Medtronic and Microport. The other authors have no conflicts of interest to declare.

\section{References}

1. Baumgartner H, Falk V, Bax JJ, et al. 2017 ESC/EACTS Guidelines for the management of valvular heart disease. Eur Heart J 2017;38:2739-91.

2. Tchetche D, Farah B, Misuraca L, et al. Cerebrovascular events post-transcatheter aortic valve replacement in a large cohort of patients: a FRANCE-2 registry substudy. JACC Cardiovasc Interv 2014;7:1138-45.

3. Auffret V, Regueiro A, Del Trigo M, et al. Predictors of Early Cerebrovascular Events in Patients With Aortic Stenosis Undergoing Transcatheter Aortic Valve Replacement. J Am Coll Cardiol 2016;68:673-84. 
4. Schmidt T, Leon MB, Mehran R, et al. Debris Heterogeneity Across Different Valve Types Captured by a Cerebral Protection System During Transcatheter Aortic Valve Replacement. JACC Cardiovasc Interv 2018;11:1262-73.

5. Nombela-Franco L, Webb JG, de Jaegere PP, et al. Timing, predictive factors, and prognostic value of cerebrovascular events in a large cohort of patients undergoing transcatheter aortic valve implantation. Circulation 2012;126:3041-53.

6. Tay EL, Gurvitch R, Wijesinghe N, et al. A high-risk period for cerebrovascular events exists after transcatheter aortic valve implantation. JACC Cardiovasc Interv 2011;4:1290-7.

7. Amat-Santos IJ, Rodes-Cabau J, Urena M, et al. Incidence, predictive factors, and prognostic value of new-onset atrial fibrillation following transcatheter aortic valve implantation. J Am Coll Cardiol 2012;59:178-88.

8. Mérie C, Kober L, Skov Olsen P, et al. Association of warfarin therapy duration after bioprosthetic aortic valve replacement with risk of mortality, thromboembolic complications, and bleeding. JAMA 2012;308:2118-25.

9. Makkar RR, Fontana G, Jilaihawi H, et al. Possible Subclinical Leaflet Thrombosis in Bioprosthetic Aortic Valves. New Engl J Med 2015;373:2015-24.

10. Fuchs A, De Backer O, Brooks M, et al. Subclinical leaflet thickening and stent frame geometry in selfexpanding transcatheter heart valves. EuroIntervention 2017;13:e1067-e1075.

11. Chakravarty T, Sondergaard L, Friedman J, et al. Subclinical leaflet thrombosis in surgical and transcatheter bioprosthetic aortic valves: an observational study. Lancet 2017;389:2383-92.

12. Sondergaard L, De Backer O, Kofoed KF, et al. Natural history of subclinical leaflet thrombosis affecting motion in bioprosthetic aortic valves. Eur Heart J 2017;38:2201-7.

13. Stortecky S, Stefanini GG, Pilgrim T, et al. Validation of the Valve Academic Research Consortium Bleeding Definition in Patients With Severe Aortic Stenosis Undergoing Transcatheter Aortic Valve Implantation. J Am Heart Assoc 2015;4:e002135.

14. Généreux P, Cohen DJ, Williams MR, et al. Bleeding complications after surgical aortic valve replacement compared with transcatheter aortic valve replacement: insights from the PARTNER I Trial (Placement of Aortic Transcatheter Valve). J Am Coll Cardiol 2014;63:1100-9.

15. Généreux P, Head SJ, Van Mieghem NM, et al. Clinical outcomes after transcatheter aortic valve replacement using valve academic research consortium definitions: a weighted meta-analysis of 3,519 patients from 16 studies. J Am Coll Cardiol 2012;59:2317-26.

16. Piccolo R, Pilgrim T, Franzone A, et al. Frequency, Timing, and Impact of Access-Site and Non-AccessSite Bleeding on Mortality Among Patients Undergoing Transcatheter Aortic Valve Replacement. JACC Cardiovasc Interv 2017;10:1436-46.

17. Jensen CJ, Naber CK, Urban P, et al. Two-year outcomes of high bleeding risk patients with acute coronary syndrome after Biolimus A9 polymer-free drug-coated stents: a LEADERS FREE substudy. EuroIntervention 2018;13:1946-9.

18. Nishimura RA, Otto CM, Bonow RO, et al. 2017 AHA/ ACC Focused Update of the 2014 AHA/ACC Guideline for the Management of Patients With Valvular Heart Disease: A Report of the American College of Cardiology/ American Heart Association Task Force on Clinical Practice Guidelines. Circulation 2017;135:e1159-e1195.

19. Cerrato E, Nombela-Franco L, Nazif TM, et al. Evaluation of current practices in transcatheter aortic valve implantation: The WRITTEN (WoRldwIde TAVI ExperieNce) survey. Int J Cardiol 2017;228:640-7.

20. Noble S, Asgar A, Cartier R, et al. Anatomo-pathological analysis after CoreValve Revalving system implantation. EuroIntervention 2009;5:78-85.

21. Ussia GP, Scarabelli M, Mule M, et al. Dual antiplatelet therapy versus aspirin alone in patients undergoing transcatheter aortic valve implantation. Am J Cardiol 2011;108:1772-6.

22. Stabile E, Pucciarelli A, Cota L, et al. SAT-TAVI (single antiplatelet therapy for TAVI) study: a pilot randomized study comparing double to single antiplatelet therapy for transcatheter aortic valve implantation. Int J Cardiol 2014;174:624-7.

23. Rodés-Cabau J, Masson JB, Welsh RC, et al. Aspirin Versus Aspirin Plus Clopidogrel as Antithrombotic Treatment Following Transcatheter Aortic Valve Replacement With a Balloon-Expandable Valve: The ARTE (Aspirin Versus Aspirin + Clopidogrel Following Transcatheter Aortic Valve Implantation) Randomized Clinical Trial. JACC Cardiovasc Interv 2017;10:1357-65.

24. Maes F, Stabile E, Ussia GP, et al. Meta-Analysis Comparing Single Versus Dual Antiplatelet Therapy Following Transcatheter Aortic Valve Implantation. Am J Cardiol 2018;122:310-5.

25. Nijenhuis VJ, Bennaghmouch N, Hassell M, et al. Rationale and design of POPular-TAVI: antiPlatelet 
therapy fOr Patients undergoing Transcatheter Aortic Valve Implantation. Am Heart J 2016;173:77-85.

26. Abdul-Jawad Altisent O, Durand E, Munoz-Garcia AJ, et al. Warfarin and Antiplatelet Therapy Versus Warfarin Alone for Treating Patients With Atrial Fibrillation Undergoing Transcatheter Aortic Valve Replacement. JACC Cardiovasc Interv 2016;9:1706-17.

27. Overtchouk P, Guedeney P, Rouanet S, et al. Long-Term Mortality and Early Valve Dysfunction According to Anticoagulation Use: The FRANCE-TAVI registry. J Am Coll Cardiol 2018. [Epub ahead of print].

28. Dentali F, Riva N, Crowther M, et al. Efficacy and safety of the novel oral anticoagulants in atrial fibrillation: a

Cite this article as: McHugh F, Ahmed K, Neylon A, Sharif F, Mylotte D. Antithrombotic management after transcatheter aortic valve implantation. J Thorac Dis 2018;10(Suppl 30):S3620-S3628. doi: 10.21037/jtd.2018.10.59 systematic review and meta-analysis of the literature. Circulation 2012;126:2381-91.

29. Seeger J, Gonska B, Rodewald C, et al. Apixaban in Patients With Atrial Fibrillation After Transfemoral Aortic Valve Replacement. JACC Cardiovasc Interv 2017;10:66-74.

30. Attinger-Toller A, Maisano F, Senn O, et al. "One-Stop Shop": Safety of Combining Transcatheter Aortic Valve Replacement and Left Atrial Appendage Occlusion. JACC Cardiovasc Interv 2016;9:1487-95.

31. Gross L, Jochheim D, Nitschke T, et al. Platelet Reactivity and Early Outcomes after Transfemoral Aortic Valve Implantation. Thromb Haemost 2018;118:1832-8. 